\title{
Preface to the Festschrift in Honor of Shu Chien's 80th Birthday
}

\author{
LARRY V. MCINTIRE \\ Coulter Department of Biomedical Engineering, Georgia Institute of Technology and Emory University, Atlanta, GA, USA \\ (Received 15 November 2011; accepted 16 November 2011; published online 24 November 2011)
}

Associate Editor Edward Guo oversaw the review of this article.

When one examines all of the accomplishments of Shu Chien in his first 80 years, the number and scope of these are truly amazing. This volume focuses on Shu's seminal impact on the rapidly growing field of "mechanobiology", but when looking over the more than 500 peer reviewed publications in his bibliography one can easily identify many other areas where Shu's scientific contributions have provided the rich foundation. These include examples such as vascular physiology and the role of blood rheology in human pathologies including sickle cell anemia.

Shu Chien has also made tremendous contributions to the development and federal funding of the biological sciences and biomedical engineering. He has served as President of the American Physiological Society, the Federation of American Societies for Experimental Biology (FASEB), the American Institute of Medical and Biological Engineering (AIMBE), and the Biomedical Engineering Society. While President of AIMBE, Shu was instrumental in getting the National Institute of Biomedical Imaging and Bioengineering established within the National Institutes of Health. He has the stature, personality, and integrity to work exceptionally well within the political systems of federal funding agencies and to make everyone feel they won (while in reality Shu's point of view prevailed). In recognition of all of his achievements, Shu has been elected to the Institute of Medicine, the National Academy of Engineering, the National Academy of Sciences, the Academia Sinica, and as a foreign member of the Chinese Academy of Sciences.

Shu Chien was one of the first to recognize the important coupling between cell biology and biomechanics. His early series of papers in Science in 1967 with Gregersen demonstrated the importance of cell membrane properties and intracellular structures on the ability of red cells to traverse small pores. These were followed by several decades of investigations with long time collaborators Richard Skalak and Shelly Weinbaum that probed the biomechanical basis for leukocyte rheology and the generation of atherosclerosis and other vascular pathologies. More recently Shu has taken advantage of the rapid advances in molecular biology, genomics, systems biology, and proteomics to further our understanding of the detailed interacting pathways of mechanotransduction in endothelial cells, osteoclast-like cells and progenitor cells. As an example, in a series of recent papers in the Proceedings of the National Academy of Sciences USA in 2010 and 2011, Shu has probed the role of specific microRNAs in flow regulation of endothelial cell biology. We are getting very close to a real understanding of the molecular basis of mechanotransduction and the role of these pathways in human physiology and pathophysiology. The papers in this Volume demonstrate the range of important problems in human health that are regulated to a large extent by mechanobiology. The authors are colleagues of Shu, many of whom were his students and fellows.

With each decade, Shu seems to accelerate the pace of his research and contributions. We can hardly wait to read the next series of papers coming from his group. Our best wishes to you and your wonderful wife K.C. on your 80th birthday and thank you for all of the scientific advances and professional leadership you have given us and will continue to give us. We are all in your debt.

Address correspondence to Larry V. McIntire, Coulter Department of Biomedical Engineering, Georgia Institute of Technology and Emory University, Atlanta, GA, USA. Electronic mail: larry.mcintire@bme.gatech.edu 